Communications in Physics, Vol. 24, No. 1 (2014), pp. 35-43

\title{
HEAVY DIQUARK EFFECTIVE THEORY AND SUPERSYMMETRY OF HADRONS CONTAINING A SINGLE HEAVY QUARK
}

\author{
NGUYEN AI VIET \\ Information Technology Institute, Vietnam National University, Hanoi, Vietnam \\ E-mail: naviet@vnu.edu.vn
}

Received 25 December 2013

Accepted for publication 22 February 2014

\begin{abstract}
A new supersymmetry is proposed for hadrons containing a single heavy quark. This supersymmetry is based on a new approximation to those hadrons, which we would consider as a further step beyond the spectator light diquark model of baryons. The heavy diquark effective theory is constructed by the techniques introduced in a different context by Georgi and Wise [1] and by Carone [2]. This theory can be incorporated into a supersymmetric theory together with Heavy Quark Effective Theory, and leads to a common universal Isgur-Wise function for mesons and baryons.
\end{abstract}

Keywords: heavy quark, diquark, heavy quark effective theory, supersymmetry.

\section{INTRODUCTION}

The hadrons containing one heavy quark turn out to have new symmetries, which are not manifest in QCD [3,4]. So their properties are constrained to a simpler description, the framework of the heavy quark effective theory [5-10] (HQET), where the mass of the heavy quark $M_{Q}$ is taken to infinity, with its velocity held fixed. In HQET, the heavy quark represents the kinematics of 1HQ-hadrons and is integrated out of dynamics as a very strong static color source or Wilson line, while the light degrees of freedom carry all the dynamics inside those hadrons. So the dynamics of 1HQ hadrons is independent of the mass and spin of the heavy quark. This leads to a $S U\left(2 N_{f}\right)$ symmetry for each heavy quark velocity ( $N_{f}$ is the number of heavy flavors). Hence the long wavelength of light degrees of freedom in such hadrons are related by this symmetry, which gives relations between the weak form factors. These relations express the weak form factors in term of a single reduced form factor, sometimes called the Isgur-Wise function, which maybe crucial in a reliable extraction of the Cabbibo-Kobayashi-Maskawa matrix elements from the experimental data.

To push the program further to actually calculate the form factors, it is necessary to include some dynamics. Even at this stage, some more dynamical symmetries are useful to simplify the problem to some calculable models. Lichtenberg [12] has proposed some supersymmetries of hadrons, based on the existence of the light diquark in 1HQ-baryons. These supersymmetries have 
simplified the three-body problem of baryons to the two-body problem of mesons. (Hereafter we shall refer to this type of supersymmetry as LSUSY). In this paper we shall pay special attention to an alternative one: the heavy supersymmetry (HSUSY).

\section{LIGHT DIQUARK AND LIGHT SUPERSYMMETRY BETWEEN MESONS AND BARYONS}

The calculations of heavy baryons are simplified greatly by the use of the quark-diquark model of baryons (see [13] for the latest review on diquark). This model turns out to work rather well for the exclusive reactions [14-16] using the fact that heavy baryons can be assumed to consist of a heavy quark and a light diquark. During the weak decays of the heavy quark the light diquark is assumed to be a spectator. In other words, the light degrees of freedom are assumed to be integrated into a pointlike diquark. So $1 \mathrm{HQ}$-baryons are treated as a two-body system like mesons. More simplifications can be achieved by observing that since the light diquark and the antiquark in 1HQ-baryons and in their meson superpartners have the same color index, the spin and velocity-dependent corrections to the dynamics are negligible beside the very heavy strong static color force from the heavy quark. The calculations of Hussain, Körner and Migneron [17] proved that the decay rates of baryons and mesons are equal as a consequence of the spectator model of light degrees of freedom. This has given an indication of the existence of supersymmetry in the model they used. Namely, if the antiquark was replaced by a diquark with the same colour the dynamics inside hadrons will remain unchanged. This supersymmetry between light diquark and antiquark leads to the LSUSY between mesons and baryons. For light hadrons LSUSY should be broken badly [18-21] as the differences between diquark and antiquark are not negligible at that scale. So LSUSY is proposed to be applied for 1HQ-hadrons [12]. Here the mass of light quarks is to be compared to the mass of the heavy quark. In such a meaning this supersymmetry would be very good (comparing with the usual chiral symmetry where the quark masses are compared to the scale of QCD $\Lambda_{Q C D}<<M_{Q}$ ). However there are some arguments against LSUSY, not on the diquark-antiquark supersymmetry but on the existence of the light diquark itself

i) The existence of a light diquark in 1HQ-hadrons is doubtful. The gluons that the heavy quark exchanges with light degrees of freedom are soft only for the heavy quark but are extremely hard for the light degrees of freedom (as their momenta square are of order $\left.M_{Q} \cdot \Lambda_{Q C D}\right)$. Suppose that at some moment there exists some pointlike light diquark. The dynamics also contains hard gluon exchanges which have enough energy to break the binding of the light diquark. If we decide to integrate hard gluons into the diquark to have a strong binding of diquark and a weak interaction regime with the heavy quark, we should have a very large diquark and therefore could not approximate it by a local field.

ii) The situation is even worse for a vector diquark. As the heavy quark could hardly rotate around the vector diquark, the centrifugal force would destroy the rotating vector diquark. So the light diquark picture is a poorer approximation for $\Sigma$ and $\Xi$ baryons than it is for $\Lambda$ ones.

iii) The calculations of Fleck et al. [22] have indicated that in the baryons containing a single heavy quark the correlation of $q q$ is always much weaker than the one of $Q q$. 
It has been also shown that the heavier the baryon is, the better is the heavy diquark approximation.

iv) Analogously, in atomic physics the $H e$ atom can be well approximated as a system of an electron and a $\mathrm{He}^{+}$ion. It can hardly be treated as a $\mathrm{He}^{++}$ion and a bound pair of two electrons.

\section{HEAVY DIQUARK AND HEAVY SUPERSYMMETRY BETWEEN MESONS AND BARYONS}

Fortunately, if it is so, the heavy diquark picture will be a good approximation to 1HQbaryons and it will provide a new simplification: the heavy supersymmetry (HSUSY). This supersymmetry in some sense is closer to the heavy quark symmetries. The antidiquark $\bar{D}_{a, i}=\left(\bar{Q} \bar{q}_{a}\right)_{i}$ and the quark $Q_{i}$ have the same color i (the flavour index $a$ denotes the light flavours u,d,s) so represent the same static color source. As the spin of this color source is irrelevant by spin decoupling the dynamics of the remaining light degrees of freedom in 1HQ-hadrons should be the same and perfectly supersymmetric. This picture is an approximation beyond the spectator model of baryons. One light quark does not want to be spectator anymore and approaches closer to the heavy quark to form a heavy diquark. This heavy diquark has integrated hard gluons into itself to build a very strong binding. So the heavy diquark is extremely stable while the rest light quark remains spectator. As inside the diquark the spin orientation of the heavy quark is irrelevant there is a good symmetry between scalar and vector diquarks. As we shall see below, it is possible to demonstrate the spin decoupling of diquarks in an effective heavy diquark theory. It is possible to derive the low-energy QCD, which contains the diquark fields as collective coordinates. Such a low energy QCD can be derived from the first principles of QCD (For instance as in the works done by Cahill [23] and Reinhardt [24] who developed an original idea of Kleinert [25] on bilocal operators, which have been interpreted as diquarks in some approximation). Here we shall take only the lowest terms. Namely it is the minimal $S U(3)_{\text {colour }}$ theory of a scalar field and a vector field coupled to the gluon field through the usual Yukawa-coupling in the covariant derivative. We shall see that in the infinity mass limit this theory will also lead to an effective diquark theory, which is perfectly parallel with HQET in a supersymmetric way. Therefore we can put together scalar and vector antidiquarks into a 7-dimensional supermultiplet together with the quark. This supermultiplet forms a spinor representation of the supergroup SU(1/6):

$$
S^{i}=\left(\begin{array}{c}
Q^{i} \\
\bar{D}_{0 a}^{i} \\
\bar{D}_{3 a}^{i}
\end{array}\right)
$$

where $D_{0 a}^{i}$ and $D_{3 a}^{i}$ denote scalar and vector diquarks with the color $i$ and the light flavor $a$. For the moment we shall omit the spin indexes.

The supergroup SU(1/6) has the following decomposition:

$$
S U(1 / 6) \supset U(1) \times S U(3)_{D_{0}} \times S U(3)_{D_{3}}
$$

The two SU(3)-groups given in the above decomposition mix flavors of anti-diquarks. We shall differentiate between them by an index $A$, where $A=D_{0}, D_{3}$. The $S U(3)_{A}$ group is generated by GellMann-matrices $\lambda_{A, p}, p=1, \ldots, 8$. The columm and row indices of $\lambda$-matrices will be denoted by $\dot{i}, \dot{j}, \dot{k}$ and $i, j, k$ respectively. In the $7 \times 7$ representation of the graded Lie algebra $S U(1 / 6)$ we 
put the elements of the Lie algebras $U(1)$ and the two $S U(3)$ into the boxes lying on the diagonal of the $7 \times 7$ matrix as follows:

$$
B=\left(\begin{array}{ccc}
U(1) & 0 & 0 \\
0 & \left(S U(3)_{D_{0}}\right)_{i}^{\dot{k}} & 0 \\
0 & 0 & \left(S U(3)_{D_{3}}\right)_{i}^{\dot{k}}
\end{array}\right)
$$

The general element of the GLA $S U(1 / 6)$ has the following form:

$$
G=\left(\begin{array}{ccc}
b_{1} & f_{1}^{+} & f_{2}^{+} \\
f_{1} & b_{2} & b_{4}^{+} \\
f_{2} & b_{4} & b_{3}
\end{array}\right)
$$

where $b_{1}$ is a number, $b_{2}$ and $b_{3}$ are $\mathrm{SU}(3)$-matrices, $f_{1}, f_{2}$ and $b_{4}$ are arbitrary matrices. Beside the generators given in the equation (3) the Bose sector of the GLA $S U(1 / 6)$ also contains the generators which mix the flavors of the scalar and vector antidiquark. We have denoted these matrices by $\left(b_{4}\right)_{i}^{\dot{k}}$ and by $\left(b_{4}^{+}\right)_{\dot{k}}^{i}$. These matrices are spanned by Gell Mann matrices $\lambda_{4 a},(a=$ $1, \ldots 8)$ and the $3 \times 3$ unit matrix $\lambda_{4}, 0$. We have 36 even generators of the Bose sector.

The Fermi sector contains 12 odd generators: $\left(f_{1,2}\right)^{\dot{k}}$ and $\left(f_{1,2}\right)_{k}$ mixing the quark with antidiquarks. Here we ignore the spin orientations of the quark and the diquarks. The heavy quark spin symmetry can be incorporated in a larger supersymmetry like $S U(2 / 12)$.

We shall denote the 48 generators defined above by $\left(\beta_{I}\right)_{P}^{Q}$. Sometimes the hermitian generators can be used instead of these non-hermitian ones and are defined as follows:

$$
\begin{aligned}
& b_{4}^{1}=b_{4}+b_{4}^{+} \\
& b_{4}^{2}=i\left(b_{4}-b_{4}^{+}\right) \\
& f_{1,2}^{1}=f_{1,2}+f_{1,2}^{+} \\
& f_{1,2}^{2}=i\left(f_{1,2}-f_{1,2}^{+}\right)
\end{aligned}
$$

The group elements of the $\mathrm{SU}(1 / 6)$ are given by:

$$
g=\exp \left(i \omega^{I} \cdot \beta_{I}\right)
$$

The parameters $\omega$ are real if we use the hermitian generators $\beta_{I}$.

There are some rules which will be useful for us later to construct the SU(1/6)-invariant and covariant quantities: (see [26] for a general analysis of $S U(\mathrm{~m} / n)$-supergroups and rigorous proofs)

i) If $\tilde{S}$ is a contravariant $\mathrm{SU}(1 / 6)$-spinor and $U$ is a covariant one then the product $\tilde{S} . U$ is invariant.

ii)The quantity $\tilde{S} \beta_{I} U$ is a $\mathrm{SU}(1 / 6)$-covariant vector.

The heavy diquark picture for 1HQ-baryons has a very attractive feature that we can construct an effective theory for heavy diquark by the same route as HQET. We are in a lucky situation as the techniques required to do this have been already worked out by Georgi and Wise [1] and by Carone [2], who have constructed effective theories for heavy scalars and for heavy vectors in a different context.

Let us now construct the effective theory for heavy diquarks. The Foldy-Wouthuysen transformation of heavy quark takes the following form:

$$
Q_{v}^{i}(x)=\exp \left(i M_{Q} \not \partial v_{\mu} x^{\mu}\right) \cdot Q^{i}(x)
$$


The scalar diquark has the following Foldy-Wouthuysen transformation:

$$
\bar{D}_{0 a, v}^{i}(x)=\exp \left(i M_{D_{0}} v_{\mu} x^{\mu}\right) \cdot \bar{D}_{0 a}^{i}(x)
$$

The vector diquark has the following Foldy-Wouthuysen transformation:

$$
\bar{D}_{3 a, v}^{i}(x)=\exp \left(i M_{D_{3}} v_{\mu} x^{\mu}\right) \cdot \bar{D}_{3 a}^{i}(x)
$$

So in a supersymmetric form the above Foldy-Wouthuysen transformation can be written as follows:

$$
S_{v}^{i}(x)=\exp \left(-i \mathbf{M} \quad N v_{\mu} x^{\mu}\right) \cdot S^{i}(x)
$$

where

$$
\begin{gathered}
W=\boldsymbol{\Gamma}_{\mu} \cdot v^{\mu} \\
\boldsymbol{\Gamma}_{\mu}=\left(\begin{array}{ccc}
\gamma_{\mu} & 0 & 0 \\
0 & v_{\mu} & 0 \\
0 & 0 & v_{\mu}
\end{array}\right) \\
\mathbf{M}=\left(\begin{array}{ccc}
M_{Q} & 0 & 0 \\
0 & M_{D_{0}} & 0 \\
0 & 0 & M_{D_{3}}
\end{array}\right)
\end{gathered}
$$

and

$$
v^{2}=v_{\mu} \cdot v^{\mu}=1
$$

The matrices $M$ and $\Gamma$ do not act on the SU(1/6)-indices but only on the spin indices of quark and antidiquarks in a reducible representation. All terms that refer to the creation and annihilation of heavy particles are ignored when taking $M_{Q, D_{0}, D_{3}} \longrightarrow \infty$ with the velocity $v_{\mu}$ held fixed, as these processes are suppressed in the limit we are considering. The leading term in the effective Lagrangian takes the form

$$
L_{v}=\frac{i}{2}\left(\bar{Q}_{v} v_{\mu} \stackrel{\leftrightarrow}{D}^{\mu} Q_{v}+2 M_{D} \bar{D}_{0 v}^{*} v_{\mu} \stackrel{\leftrightarrow}{D}^{\mu} \bar{D}_{0 v}-2 M_{D_{3}} g_{\alpha \beta} \bar{D}_{3 v}^{\alpha *} v_{\mu} \stackrel{\leftrightarrow}{D}^{\mu} \bar{D}_{3 v}^{\beta}\right)
$$

The color and flavor indices are contracted properly. To write this Lagrangian in a supersymmetric form let us define

$$
\bar{S}_{v}^{i}=S_{v}^{i *} \Gamma_{0}=S_{v}^{i *}\left(\begin{array}{ccc}
\gamma_{0} & 0 & 0 \\
0 & 1 & 0 \\
0 & 0 & -g
\end{array}\right)
$$

where $g$ is the metric matrix. So

$$
L_{v}=\frac{i}{2}\left(\bar{S}_{v} \tilde{M} v_{\mu} \stackrel{\leftrightarrow}{D}^{\mu} S_{v}\right)
$$

where

$$
\tilde{M}=\left(\begin{array}{ccc}
1 & 0 & 0 \\
0 & 2 M_{D_{0}} & 0 \\
0 & 0 & 2 M_{D_{3}}
\end{array}\right)
$$


We can redefine diquarks so that the $\tilde{M}$-matrix is unit by the following normalization

$$
D_{a}^{i} \longleftarrow \frac{D_{a}^{i}}{\sqrt{2 M_{D}}}
$$

Hence we have an effective Lagrangian

$$
L_{v}=\frac{i}{2}\left(\bar{S}_{v} v_{\mu} \stackrel{\leftrightarrow}{D}^{\mu} S_{v}\right)
$$

which is $S U(1 / 6)$-invariant manifestly under the following transformations:

$$
\delta S_{v}=i \omega_{I} \beta^{I} S_{v}
$$

Let us notice that the supermultiplet is decoupled from the gluon field by the gauge fixing condition:

$$
v_{\mu} \cdot A^{\mu}=0
$$

Using the rules mentioned above we have the $S U(1 / 6)$-covariant vector currents

$$
J_{\mu}^{I}(1,2)=\bar{S}_{v 1} \tilde{\boldsymbol{\Gamma}}_{\mu} \beta^{I} S_{v 2}
$$

where

$$
\tilde{\boldsymbol{\Gamma}}_{\mu}=\left(\begin{array}{ccc}
\gamma_{\mu} & 0 & 0 \\
0 & \overleftrightarrow{\partial}_{\mu} & 0 \\
0 & 0 & \overleftrightarrow{\partial}_{\mu}
\end{array}\right)
$$

$S_{v 1}$ and $S_{v 2}$ denote different heavy flavored supermultiplet (They can take any of the flavors c,b,t). The supermultiplet of 1HQ-hadrons can be given by the tensor product of a light antiquark with the supermultiplet $S$ :

$$
H_{b}=S^{i} \cdot \bar{q}_{i b}=\left(\begin{array}{c}
Q^{i} \cdot \bar{q}_{i b} \\
\bar{D}_{a}^{i} \bar{q}_{i b}
\end{array}\right)
$$

This supermultiplet contains mesons and antibaryons. The light antiquark is unchanged under the HSUSY transformations of $\mathrm{SU}(1 / 6)$. So $H_{b}$ is a SU(1/6)-covariant spinor.

The matrix elements of the above current between the supermultiplet $H_{1}$ and $H_{2}$ will be $S U(1 / 6)$-invariant. Therefore by the Wigner-Eckhart theorem all these matrix elements are determined in terms of a single reduced matrix element. This is the Isgur-Wise function.

For example, if we want to calculate the matrix elements between any two states $\Psi_{1 P}\left(v^{\prime}\right)$ and $\Psi_{2 Q}(v)$, which are antibaryons or mesons of the supermultiplets $H_{1}$ or $H_{2}(n, l$ denote the compnents of the hadron supermultiplets). The matrix elements can now be computed as follows:

$$
\left\langle\Psi_{1 P}\left(v^{\prime}\right)\left|J_{\mu I}\left(v^{\prime}, v\right)\right| \Psi_{2 Q}(v)\right\rangle=-\xi\left(v^{\prime} v\right) \cdot \operatorname{Tr}\left(\bar{\Psi}_{1 P} \tilde{\Gamma}_{\mu}\left(v^{\prime}, v\right)\left(\beta_{I}\right)_{P}^{Q} \Psi_{2 Q}\right)
$$

In the expression above we can replace $i \vec{\partial}_{\mu}$ and $-i \overleftarrow{\partial}_{\mu}$ by $M_{2} \cdot v_{\mu}$ and $M_{1} \cdot v_{\mu}^{\prime}$ in $\tilde{\boldsymbol{\Gamma}}_{\mu}$ as the heavy supermultiplet is nearly on the mass shell.

The heavy supermultiplet satisfies the velocity selection rule:

$$
N \cdot S=S .
$$

Let us work out the trace formula (26) for the matrix elements of the weak current $J_{\mu}^{V-A}$ between the hadron states. To do so we should follow the steps: 
i) To give an ansatz for the wave functions of the mesons $\mathrm{P}, 0^{-}$and $\mathrm{V}, 1^{+}$and of the baryons $\Lambda(\Xi), \frac{1}{2}^{+}, \Sigma(\Omega), \frac{1}{2}^{+}$and $\Sigma^{*}\left(\Omega^{*}\right), \frac{3}{2}^{+}$.

ii) To substitute the ansatz and the expressions (3)-(5) and (22) for $\beta_{I}$ and $\tilde{\Gamma}_{\mu}$ into the formula (26) then work out the trace.

Here we present only the main results:

The ansatz for the mesons'wave functions is given as follows

$$
\begin{array}{r}
P=\left(\begin{array}{c}
\sqrt{M_{P}} \gamma_{5}(1-\not \partial) / 2 \\
0 \\
0
\end{array}\right) \\
V=\left(\begin{array}{c}
\sqrt{M_{V}} \not k(1-\not \partial) / 2 \\
0 \\
0
\end{array}\right)
\end{array}
$$

where $\epsilon$ denotes the polarization vector of the vector meson $1^{+}$

The ansatz for the baryons' wave functions is given as follows

$$
\Lambda=\left(\begin{array}{c}
0 \\
u^{T} C \cdot \sqrt{2 M_{\Lambda}} \\
0
\end{array}\right)
$$

where the spinor $u$ satisfies the velocity selection rule

$$
\not p u=u
$$

and the unconventional normalization:

$$
\bar{u} u=1
$$

$\mathrm{C}$ is the charge conjungation matrix.

$$
\begin{gathered}
\Sigma=\frac{1}{\sqrt{6 M_{\Sigma}}}\left(\begin{array}{c}
0 \\
0 \\
u^{T} C \sigma^{\mu \nu} v_{\nu} \gamma_{5}
\end{array}\right) \\
\Sigma^{*}=\frac{1}{\sqrt{2 M_{\Sigma^{*}}}}\left(\begin{array}{c}
0 \\
0 \\
u^{\mu T} C
\end{array}\right)
\end{gathered}
$$

$u^{\mu}$ is a Rarita-Schwinger spinor satisfying the equations:

$$
\begin{gathered}
-\bar{u}_{\mu} u^{\mu}=1 \\
\not v u^{\mu}=u^{\mu}
\end{gathered}
$$

We can impose the gauge fixing conditions:

$$
v_{\mu} u^{\mu}=0
$$

and

$$
\gamma_{\mu} u^{\mu}=0
$$

The formula (26) gives the following matrix elements of the weak currents:

$$
<P_{Q_{1}}\left(v^{\prime}\right)\left|J_{\mu}^{V-A}\right| P_{Q_{2}}(v)>=\xi\left(v^{\prime} v\right)\left(v_{\mu}+v_{\mu}^{\prime}\right) \sqrt{M_{P_{1}} \cdot M_{P_{2}}}
$$




$$
\begin{aligned}
<V_{Q_{1}}\left(v^{\prime}\right)\left|J_{\mu}^{V-A}\right| V_{Q_{2}}(v)>= & -\xi\left(v^{\prime} v\right)\left[\left(\epsilon_{1}^{*} \epsilon_{2}\right)\left(v_{\mu}+v_{\mu}^{\prime}\right)-\left(\epsilon^{*} v\right) \epsilon_{2 \mu}\right. \\
\left.-\left(\epsilon_{2} v\right) \epsilon_{1 \mu}\right] \sqrt{M_{V_{1}} \cdot M_{V_{2}}} & \bar{r}_{\Lambda_{Q_{1}}\left(v^{\prime}\right)\left|J_{\mu}^{V-A}\right| \Lambda_{Q_{2}}(v)>=}\left(v^{\prime} v\right) \frac{1}{2}\left(v_{\mu}+v_{\mu}^{\prime}\right) \bar{u}_{1} u_{2} \sqrt{M_{\Lambda_{1}} \cdot M_{\Lambda_{2}}} \\
<\Sigma_{Q_{1}}\left(v^{\prime}\right)\left|J_{\mu}^{V-A}\right| \Sigma_{Q_{2}}(v)>= & \frac{1}{6} \xi\left(v^{\prime} v\right)\left[\left(2+v^{\prime} v\right)\left(v_{\mu}^{\prime}+v_{\mu}\right) \bar{u}_{1} u_{2}\right. \\
& \left.+\left(1+v^{\prime} v\right) \bar{u}_{1}\left(2 \gamma_{\mu}-v_{\mu}-v_{\mu}^{\prime}\right) u\right] \sqrt{M_{\Sigma_{1}} \cdot M_{\Sigma_{2}}} \\
<\Sigma_{Q_{1}}^{*}\left(v^{\prime}\right)\left|J_{\mu}^{V-A}\right| \Sigma_{Q_{2}}^{*}(v)>= & \frac{1}{2} \xi\left(v^{\prime} v\right)\left[-\left(v_{\mu}^{\prime}+v_{\mu}\right) \bar{u}_{1 \mu} u_{2}^{\mu}+\bar{u}_{1 \mu} v_{\nu}^{\prime} u_{2}^{\nu}\right. \\
& \left.+v_{\nu} \bar{u}_{1}^{\nu} u_{\mu}\right] \sqrt{M_{\Sigma_{1}^{*}} \cdot M_{\Sigma_{2}^{*}}} \\
<\Sigma_{Q_{1}}\left(v^{\prime}\right)\left|J_{\mu}^{V-A}\right| \Sigma_{Q_{2}}^{*}(v)>= & \frac{i}{2 \sqrt{3}} \xi\left(v^{\prime} v\right)\left[\left(1+v^{\prime} v\right) \bar{u}_{1} \gamma_{5} u_{2}^{\mu}\right. \\
& \left.+\bar{u}_{1} \gamma_{5}\left(\gamma_{\mu}-v_{\mu}\right) v_{\nu}^{\prime} u_{2}^{\nu}\right] \sqrt{M_{\Sigma_{1}} \cdot M_{\Sigma_{2}}}
\end{aligned}
$$

We can also compute the light flavour changing matrix elements, which lead to the same reduced form factor $\xi\left(v^{\prime} v\right)$. The matrix elements between mesonic and baryonic states vanish because the matrix $\tilde{\Gamma}_{\mu}$ is diagonal. More details on these matrix elements will be given elsewhere.

It is worthy stressing here that new supersymmetry and new model presented above are not alternative of the LSUSY and the light diquark model. Both of them are dynamical symmetries based on certain approximations to baryons. In some meaning these supersymmetries are similar to the one of Iachello et al. [27] in nuclear physics for heavy nuclei.

\section{CONCLUSION}

In this paper, we have proposed the heavy diquark model for baryons to go a step beyond the spectator model of baryons. In this model one light quark approaches the heavy quark closer than the other light one and form a heavy diquark. The heavy diquark has a better chance to survive the gluon exchanges with the remaining quark than the light diquark does. So, the last could be approximated very well as a spectator The picture is similar to the three-body model of the helium atom [28], which can be approximated in a good approximation as a system of the helium ion and an electron.

We have constructed a supersymmetric effective model for heavy quark and heavy diquark. In this supersymmetric model the matrix elements of weak currents are characterized by one universal Isgur-Wise function and the weak formfactors of baryons collapse to the one of mesons. We can also combine the heavy quark spin symmetry with the $\mathrm{SU}(1 / 6)$ supersymmetry and put all spin components of the heavy quark and heavy antidiquark together into a 14-dimensional spinor of the supergroup $S U(2 / 12)$. Combining with the heavy flavour symmetry $S U(3)$ of Isgur and Wise the total symmetry of the "heavy world" is indeed tremendous with the supersymmetry $S U(6 / 36)$. This is the largest symmetry we know about this world. So we come to the conclusion that the set of 1 HQ-hadrons is governed by a simpler law than we thought.

The research on this topic currently has been carried further in two directions. In the first one, we model the baryons as a classical three-body system of quarks. Between the quarks, we postulate various confining interaction potentials. By a computer simulation program written in 
Mathematica, we can trace the coordinates of the quarks and calculate the average distance between the quarks. In the limit of one heavy quark with various choices of the interaction parameters, one can see how good an approximation the HSUSY is from the dynamical viewpoint [29]. In the second direction, we can analyze the Particle Data to compare the consequences of LSUSY and HSUSY with experimental data.

\section{ACKNOWLEDGMENT}

I would like to thank Professors Dao Vong Duc and Nguyen Thi Hong for their elegant formulation on gradded Lie algebra, which simplifies this work in a great deal. Thanks are also due to Dr. Le Viet Dung for many useful discussions on the diquark topic.

\section{REFERENCES}

[1] H. Georgi and M. B. Wise, Phys. Lett. B 243 (1990) 279.

[2] C. D. Carone, Phys. Lett. B 253 (1991) 408.

[3] N. Isgur and M.B.Wise, Phys.Lett. B 232 ( 1989) 113.

[4] N. Isgur and M.B.Wise, Phys.Lett. B 237 (1990) 527.

[5] H. D. Politzer and M.B.Wise, Phys.Lett. B 208 (1990) 504.

[6] E. Eichten and B. Hill, Phys. Lett. B 234 (1990) 511.

[7] H. Georgi, Phys. Lett. B 240 (1990) 447.

[8] G. P. Lepage and B.A.Thacker, Nucl. Phys. B 4 ( Proc. Suppl.) (1989) 199.

[9] M.B.Voloshin and M.A.Shifman Sov.J.Nucl.Phys., 45 (1987) 292.

[10] F.Hussain,J.G.Körner, K.Schilcher, G.Thompson, and Y. L. Wu, Phys. Lett. B 249 (1990) 295.

[11] J. G. Körner and G. Thompson, Phys. Lett. B 264 (1991) 185.

[12] D. B. Lichtenberg J.Phys.: Part. Nucl. Phys. G16(1990) 1599.

[13] M.Anselmino, E.Predazzi, S.Ekelin, S.Fredriksson and D.B.Lichtenberg Diquarks Research Report ISSN 03470881, Tuleả (1992)

[14] J. G. Körner and P.Kroll, Heavy Quark Symmetry at Large Recoil: The case of baryons DESY 92-019 MZTH/91-34 WU-B 91-31 (1992)

[15] P.Kroll, B.Quadder and W.Schweiger, Nucl.Phys. B316 (1989) 373.

[16] P.Kroll, W.Schweiger and M.Schürman, Int. J. Mod. Phys. A A6 (1991) 4107.

[17] F. Hussain, J. G. Körner and R. Migneron, Phys. Lett. B 248 (1990) 406.

[18] H. Miyazawa, Prog. Theor. Phys. (Kyoto), 36 (1966) 1266.

[19] H. Miyazawa, Phys. Rev. 170 (1968) 1586.

[20] S. Cato and F. Gürsey, Il Nuovo Cimento, 86A (1985) 201.

[21] S.Cato and F.Gürsey, Il Nuovo Cimento, 99A (1988) 685.

[22] S. Fleck, B. Silvestre-Brac and J. M. Richard Phys. Rev. D38 (1987) 1519

[23] R. T. Cahill Baryon structure and diquarks FPPG-R-15-88, Invited talk at Workshop on DIQUARKS, Torino, Italy (October 1988).

[24] H.Reinhardt, Phys. Lett. B 244 (1990) 316.

[25] H.Kleinert, in : Understanding the fundamental constituents of matter, ed. A.Zichichi (Plenum, New York, 1978).

[26] Dao Vong Duc and Nguyen Thi Hong, Ann. Int. Henri Poincaré A 36(1985) 495.

[27] A. B. Balantekin, I. Bars and F. Iachello, Nucl.Phys. A 370 ( 1981) 248.

[28] T.Yamamoto and K.Kaneko, Phys. Rev. Lett. 70(13) (1993) 1928;

[29] Nguyen Ai Viet and Dang Hoang Vu, Classical computer simulation of the heavy baryon containing one heavy quark as a three-body system (under preparation) 